\title{
Intravenous clevidipine for management of hypertension
}

\author{
This article was published in the following Dove Press journal: \\ Integrated Blood Pressure Control \\ 26 June 2010 \\ Number of times this article has been viewed
}

\begin{abstract}
Alma Rivera'
Elsa Montoya ${ }^{2}$

Joseph Varon ${ }^{3}$

'Universidad Autónoma de Chihuahua, Chihuahua, México; ${ }^{2}$ Universidad de Monterrey, Nuevo León, México; ${ }^{3}$ University of Texas Health Science Center at Houston, Texas, USA
\end{abstract}

\begin{abstract}
Hypertension remains one of the most prevalent diseases affecting our society, and its complications lead the list of causes of mortality all over the world. Most efforts to control the disease are unsuccessful, failing in at least two-thirds of affected patients, despite the availability of multiple drugs for its treatment. The limited number of medications available for aggressive management of hypertensive crises has intensified the search for novel drugs that can achieve a rapid decrease in blood pressure without increasing the possible complications. Clevidipine is a novel, vasculoselective, dihydropyridine calcium channel blocker characterized by a very fast onset and offset of action. Metabolism of clevidipine does not occur in the liver or kidneys, and thus there are no restrictions to using clevidipine in patients with hepatic or renal dysfunction. This agent has been widely used to reduce blood pressure when oral therapy is not appropriate, and its use in the perioperative setting has been shown to be beneficial. This manuscript reviews the key characteristics of clevidipine and its role in the management of acute hypertension.
\end{abstract}

Keywords: clevidipine, hypertension management, acute, hypertensive crises, intravenous

\section{Introduction}

Hypertension remains one of the most prevalent diseases affecting our society, and its complications lead the list of causes of mortality all over the world. At least $1 \%$ of all hypertensive patients present to an emergency department with a hypertensive crisis at some point in their lives. ${ }^{1}$ Defined as a sudden elevation of blood pressure (BP) associated with target organ damage, hypertensive emergencies are potentially lethal. $^{2}$

The myocardium is the most commonly affected target organ, but encephalopathy, cerebrovascular accidents, acute papilledema, aortic dissection, renal failure, and acute pulmonary edema are frequently associated with severe BP elevation. ${ }^{3}$ Predisposing factors for development of a hypertensive crisis include mechanical stress and injury, endothelial damage, renin-angiotensin system activation, and oxidative stress. ${ }^{3}$ The predominant insult seems to be a cascade of humoral vasoconstrictors that leads to increased vascular resistance, generating stress and damaging the endothelium, which activates the coagulation cascade and promotes the deposition of fibrin. These processes result in ischemia, oxidative injury, and further release of vasoactive substances that perpetuate the destructive cycle, culminating in the multiple organ dysfunction that characterizes a hypertensive emergency. ${ }^{4}$ The majority of patients found to have a hypertensive emergency have been previously diagnosed with hypertension and generally have had poor BP control. ${ }^{5}$
Correspondence: Joseph Varon

2219 Dorrington St, Houston,

TX 77030-3209, USA

$\mathrm{Tel}+17136691670$

Fax + $7 \mid 3669$ |67|

Email joseph.varon@uth.tmc.edu 
Most efforts to control the condition have been unsuccessful, failing in at least two-thirds of affected patients, despite the availability of multiple drugs for the treatment of hypertension. ${ }^{2}$ Finding an adequate antihypertensive drug suitable for the individual patient, according to his or her personal characteristics (ie, race, age, etiology, associated morbidity, and concomitant therapies) and the nature of their condition (ie, chronic hypertension, acute hypertension, hypertensive emergency, or hypertensive urgency) has proven difficult to achieve over the last 40 years, leading to an increasing number of hypertensive emergencies. ${ }^{6}$

A common reason for hypertensive emergency is patient noncompliance with medical therapy. However, there are other potential causes, including stimulant intoxication (ie, cocaine, methamphetamines, phencyclidine, etc) ${ }^{7,8}$ and antihypertensive drug withdrawal syndromes (eg, from clonidine and beta-blockers). On rare occasions, patients are found to have a pheochromocytoma or another underlying condition that triggers the crisis. ${ }^{9}$

Most patients arriving in emergency departments who are found to have severe hypertension, ie, systolic BP $(\mathrm{SBP})>160 \mathrm{mmHg}$ or diastolic $\mathrm{BP}(\mathrm{DBP})>100 \mathrm{mmHg}$, do not present with acute target organ damage, and are not cases of hypertensive emergency, but do present as hypertensive urgency cases. In these patients, oral therapy may be the best choice to lower BP gradually within 24-48 hours to avoid hypoperfusion caused by an abrupt drop in BP. $^{3}$ However, patients who present as a hypertensive emergency and with target organ damage require aggressive management of their BP to limit the hypoxic injury already taking place in these organs. For this reason, rapid-acting antihypertensive agents that can be easily titrated and administered intravenously (IV) are preferred for the management of hypertensive crisis. According to the seventh report of the Joint National Committee on Prevention, Detection, Evaluation, and Treatment of High Blood Pressure (JNC 7), the aim is to bring DBP down by $10 \%-15 \%$, but by no more than $25 \%$, or at least to $110 \mathrm{mmHg}$ within 30 to 60 minutes. $^{2}$ Once achieved, the IV medication can be slowly weaned, and BP can be maintained with oral therapy. It is important to note that hypertensive emergency per se is a clinical diagnosis, and the administration of any medication should be appropriate to the clinical status of the patient, regardless of the BP values at the time..$^{10,11}$ The management of hypertension in these settings should be done in an intensive care unit and patients should undergo close monitoring to minimize the morbidity and mortality associated with abrupt reductions in BP.

A variety of IV antihypertensive drugs can be used, depending on the patient's clinical status (eg, presence of liver or kidney failure, pregnancy, or allergies), the target organ affected, and the resources available to monitor the patient adequately. Labetalol is the most commonly used agent $(32 \%)$ to manage severe hypertension, followed by metoprolol (16\%), nitroglycerin (15\%), hydralazine (15\%), nicardipine (8\%), and sodium nitroprusside (SNP, 6\%). ${ }^{12}$ Nevertheless, the use of clevidipine in these settings has been increasing since its approval in this indication by the US Food and Drug Administration (FDA) in 2008, and several trials have confirmed its efficacy and safety.

The limited number of medications available for aggressive management of hypertensive crises, eg, in the emergency department or during specialized surgical procedures, has created a need for newer drugs that provide a rapid decrease of BP without increasing the risk of complications. Accordingly, there is continuous ongoing research on antihypertensive drugs that can be easily adapted for use in hypertensive emergencies and urgencies. This manuscript reviews the key characteristics of clevidipine $\left(\right.$ Cleviprex $\left.^{\circledR}\right)$, a novel antihypertensive agent, and its role in the management of acute hypertension.

\section{Overview of clevidipine}

Clevidipine is a novel, vasculoselective calcium channel blocker (CCB) of the dihydropyridine type which induces powerful vasodilatation in arterioles. ${ }^{6,13}$ Clevidipine was designed as an oil-in-water emulsion containing egg yolk phospholipids, soybean oil, and glycerin, and excited the research community because of its selectivity and ability to reduce afterload without affecting preload. ${ }^{13,14}$ Clevidipine was the first IV antihypertensive treatment introduced to the market after 10 years of research. ${ }^{15}$ Clevedipine's effects on human vasculature were first studied in the internal mammary artery, and it was found to have a considerable vaso-relaxing effect induced by thromboxane A2 analog. ${ }^{16}$

Clevidipine is similar to felodipine in its structure but is functionally closer to nicardipine. ${ }^{17}$ It is considered unique because of its very short duration of action (ie, rapid onset and offset), achieved by the incorporation of an additional ester group in the molecule. ${ }^{17,18}$ This results in rapid hydrolysis of the molecule by blood and tissue esterases, producing the carboxylic acid metabolite $\mathrm{H}$ 152/81. Clevidipine is a racemic mixture of enantiomers, ie, $\mathrm{R}(-)$-clevidipine and $\mathrm{S}(+)$-clevidipine, both of which impact BP, but the latter contributes to a larger extent at equivalent plasma levels. ${ }^{17}$ Clevidipine has no effects on venous vascular tone and its ability to modify afterload prevents it from affecting other hemodynamic parameters, including left ventricular filling pressure and pulmonary capillary wedge pressure. ${ }^{13}$ 


\section{Pharmacokinetics and pharmacodynamics}

Immediately after IV infusion, clevidipine is hydrolyzed primarily by blood esterases into its inactive metabolites (esterases in extravascular tissue also have a moderate role), including hemiacetal esters and butyric acid, which do not have a relevant effect on any of the CYP450 enzyme. ${ }^{19}$ Approximately $99.5 \%$ of clevidipine in plasma is bound to proteins, and this binding capacity is not concentrationdependent. ${ }^{18,20,21}$ Clevidipine is a high clearance drug, whereby the kidney is responsible for the elimination of at least $60 \%$ of the drug and the remainder is eliminated in feces. ${ }^{21}$ Clevidipine goes through the first phase of its elimination during this first minute after administration, and as much as $85 \%-90 \%$ is eliminated at this time. The remainder of the drug is metabolized and has a terminal half-life of approximately 15 minutes. ${ }^{13,21}$ This unique pattern of metabolism is not affected by hepatic dysfunction, and its rapid elimination contributes to prompt recovery of normal $\mathrm{BP}^{13}$

\section{Use of clevidipine for perioperative hypertension}

Uncontrolled BP is common in the perioperative period, and antihypertensive medication is often necessary. ${ }^{22}$ This is especially the case in cardiac surgery, where any sudden increase in BP increases the risk of postoperative complications. An increase in BP means that the myocardium has to work harder, thus increasing myocardial oxygen demand, which may ultimately lead to an increase in left ventricular filling pressure, resulting in pulmonary congestion. ${ }^{23}$ Sudden increases in BP are also responsible for hemorrhage, one of the common complications in the postoperative period. ${ }^{24}$

One of the main causes of perioperative hypertension is a generalized increase in sympathetic tone that causes vasoconstriction and in turn increased BP. The ideal antihypertensive agent would produce vasodilatation efficiently and rapidly, with minimal adverse effects. The usual drugs of choice for this purpose are nitroglycerin or SNP, with $\beta$-blockers and CCBs used in a minority of patients. ${ }^{25}$ However, since the advent of clevidipine, several trials have evaluated the safety and efficacy of the drug in this setting. Preliminary research gave a glimpse of the efficacy of clevidipine, leading to two large randomized, double-blind, placebo-controlled trials, ie, ESCAPE 1 and ESCAPE 2, that evaluated the use of clevidipine in the pre- and postoperative periods, respectively. ${ }^{26,27}$

\section{ESCAPE-I in preoperative hypertension}

The ESCAPE-1 trial included 150 hypertensive patients scheduled for cardiac surgery who received either clevidipine or placebo (lipid emulsion) via peripheral or central IV infusion over 30 minutes. ${ }^{26}$ Patients were assessed with a baseline echocardiogram, laboratory screening, vital signs recording, and careful monitoring of SBP and DBP. Treatment failures were compared between the two groups to evaluate efficacy, where treatment failure was defined as an inability to achieve at least a $15 \%$ reduction in baseline SBP. Further assessments included time taken to reach target BP, change in mean arterial pressure (MAP), change in heart rate (HR), and long- and short-term adverse effects.

This clinical trial was the first to demonstrate the efficacy of clevidipine, and showed a significantly lower rate of treatment failure compared with placebo (clevidipine $7.5 \%$ versus placebo $82.7 \%, P<0.0001$ ). The safety of clevidipine was evaluated by HR changes and the rate and type of adverse effects observed. Surprisingly, both clevidipine and placebo were free of HR changes after discontinuation of infusion, and the increase in HR observed initially was not substantial. Both treatment groups had similar adverse event rates, which included pyrexia, atrial fibrillation, acute renal failure, and nausea, in descending order. All these adverse events were more frequently noted in patients on clevidipine treatment but the difference was not statistically significant. Despite some limitations, this study demonstrated that clevidipine was successful in decreasing SBP by at least 15\% from baseline in $92.5 \%$ of the cases, achieving this within a mean of six minutes after infusion. ${ }^{26}$

\section{ESCAPE-2 in postoperative hypertension}

The presence of postoperative hypertension is a factor that negatively impacts the outcome of surgery and may have adverse consequences, including increased risk of stroke, renal injury, heart failure, and postoperative bleeding. ${ }^{28}$ Despite being a highly modifiable cardiovascular risk factor, BP has been found to be extremely hard to control following cardiac surgery. After much research concerning the effects of elevated BP, it has been shown that increased SBP (essentially when isolated) is a negative predictor and risk factor for cardiovascular morbidity. ${ }^{29}$

Systolic hypertension is the most common cause of postoperative hypertension in the elderly, partially due to the increasing proportion of patients $\geq 65$ years undergoing 
cardiac surgery. ${ }^{30}$ Arteriosclerosis is a common condition in this age group, is exacerbated by hypertension, and is a contributor to increased SBP. Another comorbidity associated with increased SBP is left ventricular hypertrophy, which has been found to contribute directly to perioperative morbidity.

The need to maintain tight control of BP during and after cardiac surgery led to the initiation of the ESCAPE-2 trial to evaluate the efficacy of clevidipine in treating postoperative hypertension. ${ }^{27}$ In this trial, 110 of 206 patients recruited met the inclusion criteria and received infusions of clevidipine or placebo. The design of the study and the variables observed in this trial were the same as those in ESCAPE-1. The study demonstrated that clevidipine was significantly better than placebo in successfully treating postoperative hypertension (91.8\% success rate with clevidipine versus $20.4 \%$ with placebo, $P<0.0001)$. A decrease in SBP was achieved in a median of 5.3 minutes with clevidipine. A significantly larger decrease in MAP was observed in the clevidipine group at 2, 5, 10, and 15 minutes after the infusion (mean change in MAP $-5.7 \mathrm{mmHg}$ versus $-0.1 \mathrm{mmHg}$ in the placebo group, $P=0.0004)$. In this study, $94.7 \%$ of patients treated with clevidipine reached the target SBP, and only one patient required maximal dose titration. No reflex tachycardia was observed in the clevidipine group, and the highest median HR recorded was 93 beats per minute. Other common adverse effects reported were nausea (21.3\%), atrial fibrillation (21.3\%), and insomnia (11.5\%), all of which were observed more frequently in the clevidipine group. Clevidipine was discontinued in one patient because of atrial fibrillation, reported as a nonserious adverse effect that resolved without sequelae on the same day after discontinuing infusion of clevidipine. ${ }^{27}$

In conclusion, both ESCAPE 1 and ESCAPE 2 demonstrated that clevidipine was safe and efficacious when used in the perioperative setting, predominantly due to its rapid onset and offset of action and easy titration. ${ }^{26,27}$

\section{Clevidipine and sodium nitroprusside}

As mentioned before, the two agents most commonly used in the treatment of postoperative hypertension are nitroglycerin and SNP. Both agents are fast-acting vasodilators, but they are not very selective and cause some venodilation. Both drugs are also known to cause reflex tachycardia, rebound hypertension, and tachyphylaxis.

In a double-blind, randomized trial, Powroznyk et al compared SNP and clevidipine in the management of postoperative hypertension and monitored hemodynamic changes in patients undergoing coronary artery bypass graft surgery. ${ }^{31} \mathrm{~A}$ reason for choosing the setting of cardiac surgery was that postoperative hypertension occurs in $30 \%-50 \%$ of all patients undergoing the procedure..$^{32}$ The study recruited 39 patients of whom 30 met the inclusion criteria, and were divided in two groups. One group received clevidipine and a SNP placebo, and the other received SNP and a clevidipine placebo. Variables evaluated were the efficacy of the drug in controlling BP, the number of dose-rate adjustments necessary to achieve that goal, and the hemodynamic impact of the drug. The study results showed no relevant differences between the efficacy of clevidipine and that of SNP. There was no significant difference between the numbers of dose-rate adjustments required for each drug; however, statistically significant data were obtained from the monitoring of vital signs. The SNP group showed a significantly greater increase in HR compared with clevidipine $(P<0.0001)$. However, the propensity of SNP to induce compensatory tachycardia is well known. Changes in central venous pressure were also more accentuated with SNP, highlighting the marked selectivity of clevidipine for arterioles. The study concluded that the overall efficacy was similar for both drugs. ${ }^{31}$

\section{ECLIPSE trial}

ECLIPSE was performed to compare clevidipine with SNP, nitroglycerin, and nicardipine in the treatment of perioperative hypertension. The study involved three parallel groups where clevidipine was compared on a 1:1 basis with one of the three other drugs. ECLIPSE was conducted in 61 hospitals and included 1512 patients who met the postrandomization criteria and were scheduled for cardiac surgery. All drugs were administered IV, although nicardipine was restricted to the postoperative period because its long half-life makes it less suitable for use before and during surgery. The drugs involved in the trial were shown to need similar infusion rates and volumes to reach the target BP. ECLIPSE demonstrated that clevidipine not only achieved superior BP control when compared with the other drugs, but also showed a significant reduction in 30-day postoperative mortality compared with SNP $(4.7 \%$ versus $1.7 \%, P=0.0445)$. There were no significant differences in incidence of cerebrovascular accident, heart attack, or kidney damage. ${ }^{33}$ The use of complementary drugs, in particular $\beta$-blockers, when the primary drug failed to achieve the target was similar among the comparator drugs, except for the group treated with SNP, which had a higher use of alternative antihypertensive therapy.

Clevidipine was more effective than the comparator drugs in controlling BP, with almost half the number of 
BP excursions compared with the other agents (3.8 versus $7.8 \mathrm{mmHg} \times \mathrm{min} /$ hour$)$. Clevidipine also had the narrowest SBP range pre- and postoperatively (105-145 $\mathrm{mmHg}$ ) and during surgery (93-135 mmHg). Clevidipine was also shown to be more effective than the other study drugs in maintaining $\mathrm{BP}$ within prespecified ranges for longer periods of time, even after modifying target BP thresholds. The study concluded that clevidipine was as safe as the other three drugs for use in the perioperative setting.

\section{VELOCITY trial}

One of the most noteworthy trials performed with clevidipine was the VELOCITY study, a Phase III, open-label, single-arm, multicenter study, designed to evaluate the use of clevidipine in patients with severe hypertension. The trial included 126 patients presenting to an emergency department or admitted to an intensive care unit with persistent severe hypertension, defined as $\mathrm{SBP}>180 \mathrm{mmHg}$ and/or DBP $>115 \mathrm{mmHg}$, assessed on two consecutive occasions with an interval of at least 15 minutes in between each measurement. Patients meeting the inclusion criteria underwent a physical examination, including urinalysis, funduscopy, 12-lead ECG, and chest radiography to determine the presence of acute or chronic target organ damage including at least one of the following: abnormal funduscopy with hypertensive changes, congestive heart failure, left ventricular hypertrophy, proteinuria or hematuria, neurologic signs, positive troponin or creatine kinase, or a symptomatic coronary syndrome with ischemic ECG changes. Patients were also screened for past medical history, baseline laboratory measurements, and medication history. Clevidipine was administered and titrated according to the prescribing information and response of the patient, and after 18 hours oral therapy could be administered if needed. During the infusion, BP and HR were constantly monitored, with blood samples obtained at regular intervals and recording of any adverse events. The results revealed that $90.5 \%$ of the patients were treated with clevidipine only, meaning that other antihypertensives were unnecessary to achieve target BP. The desired BP range was achieved within the first 30 minutes after infusion in $88.9 \%$ of the patients in a median time of 10.9 minutes, and $91.3 \%$ of the patients underwent successful transition to oral therapy. The safety results were also encouraging. The median pulse was 82 beats per minute at 3 minutes, and within the immediate 30-minute period, the mean increase observed was 10 beats per minute compared with baseline. At least one adverse effect was observed in $39.7 \%$ of patients, with $8.7 \%$ considered serious. Adverse events included headache, nausea, chest discomfort, and vomiting, in descending order. During the study, clevidipine was not associated with any clinically significant changes in laboratory test results (eg, triglyceride levels) or any increased risk of renal injury. Overall, the findings of VELOCITY were consistent with those encountered in the large trials, and clevidipine was once again demonstrated to be a safe and manageable drug for treating severe hypertension. ${ }^{12}$

\section{Indications, dosage, and contraindications}

Clevidipine is indicated in patients to reduce BP when oral therapy is not feasible. ${ }^{34}$ As noted, clevidipine is administered by IV infusion via a central or peripheral line, starting with a 1-2 mg/hour infusion, doubling the dose at 90-second intervals, and then increasing the dose by less than double at intervals of 5-10 minutes. ${ }^{35}$ Treatment and amount of clevidipine needed may vary from one patient to another, because the titration of the drug is dependent on the response and the desired BP. However, the majority of patients reach their target BP at 4-6 mg/hour in less than 30 minutes after starting the infusion with clevidipine. The maximum infusion rate is usually $16 \mathrm{mg} /$ hour, although there are insufficient data available for doses $\geq 32 \mathrm{mg} / \mathrm{hour}$ or infusions $\geq 72$ hours regardless of the dose. ${ }^{36}$

Clevidipine is packaged in single-use vials of 50 and $100 \mathrm{~mL}$ with a concentration of $0.5 \mathrm{mg} / \mathrm{mL}$, and the vehicle is a $20 \%$ phospholipid emulsion. The amount of lipids provided by the infusion of clevidipine is $0.2 \mathrm{~g} / \mathrm{mL}$, representing $2 \mathrm{kcal} / \mathrm{mL}$. For this reason it is recommended that patients do not receive more than $1000 \mathrm{~mL}$ of clevidipine in 24 hours. It is not necessary to adjust the dose in patients with liver or renal failure. ${ }^{36}$

Because of its formulation, clevidipine is contraindicated in patients allergic to soybeans, soy products, eggs, or egg products. Accordingly, this drug should used with caution in patients with abnormalities of lipid metabolism, and lipid intake restriction may be required in some patients. Patients with severe aortic stenosis should also avoid using clevidipine because it can reduce myocardial oxygen delivery as a result of significant reduction in afterload. Other contraindications of clevidipine include those common to all CCBs, and longterm monitoring is advised if the patient is not converted to oral therapy after using clevidipine.

\section{Other effects of clevidipine}

Several experimental models have been used to determine the effects of CCBs on the myocardium when administered before and after an ischemic insult. Several hypotheses 
regarding the mechanism by which CCBs achieve myocardial protection have been put forward. Activation of potassium channels, increase in blood flow to the myocardium during reperfusion, and inactivation of oxygen free radicals are now well known mechanisms. It is known that both ischemia and reperfusion cause tissue damage, and calcium overload is one of the most important mechanisms of myocardial dysfunction. Intracellular calcium levels fluctuate throughout the stages of myocardial injury, and have been found to be high both during the early ischemic period and in the early reperfusion phase, contributing to severe cellular injury. The mechanisms that trigger the insult mediated by calcium are excessive myofilament activation during reoxygenation, impaired mitochondrial function followed by a reduction in the production of adenosine triphosphate and limitation of the metabolic regeneration of the myocyte, and protease activation with subsequent tissue damage. Segawa et al demonstrated the beneficial effect of clevidipine infused shortly after the initiation of ischemia and just before reperfusion, emphasizing the role of calcium in myocardial injury. ${ }^{37}$

Although not all calcium antagonists work the same way, a narrow relationship between $\mathrm{CCBs}$ and nitric oxide (NO) is evident. NO is a potent mediator of vascular tone, and has well known actions, including prevention of platelet adherence and a protective effect against superoxide anions. Moreover, impairment of the bioavailability of NO is strongly related to endothelial dysfunction, which is the common event in several cardiovascular diseases. ${ }^{38}$

In a placebo-controlled study by Gourine et al 38 pigs were subjected to a 45-minute ligation of the left anterior descending coronary artery and administered clevidipine alone, or in combination with other vasoactive mediators affecting the synthesis and regulation of NO. Variables recorded were infarct size, hemodynamic parameters (ie, MAP, HR), and endothelial-dependent responses. This study demonstrated that clevidipine was cardioprotective when administered directly in the coronary vessels during the last 10 minutes of ischemia and in the first five minutes of reperfusion. This was indicated by a significant reduction of infarct size in clevidipine-infused pigs compared with vehicle-infused pigs $(-86 \%$ in the vehicle group versus $59 \%$ in the clevidipine group, $P<0.001)$. Moreover, the cardioprotective effect of clevidipine was strongly dependent on the local availability of NO, which was expected on the basis of a previous report. ${ }^{39}$

\section{Conclusions}

Clevidipine is a CCB characterized by a very fast onset and offset of action, and its initial use was to control hypertensive emergencies. It has a unique metabolism, occurring in the blood and extravascular tissues by esterases, giving it an initial half-life of less than 3 minutes and, because its metabolites are inactive, almost null toxicity. Its metabolism is also an attribute that ranks clevidipine over other available short-acting drugs. Because its metabolism does not occur in the liver or in the kidneys, there are no restrictions to using clevidipine in patients with hepatic or renal dysfunction. Furthermore, clevidipine infusion has a cardioprotective role in compromised myocardium.

After 10 years of research and development culminating in FDA approval in 2008, clevidipine has been widely used to reduce BP when oral therapy is not appropriate. Its use in the perioperative setting, mainly in cardiac surgery, has been shown to be beneficial compared with the few agents previously used for this purpose (eg, SNP and nitroglycerin), with a shorter duration of action and a lower incidence of adverse effects.

\section{Disclosure}

AR and EM have no financial disclosures. JV has received honoraria for lectures from PDL Pharmaceuticals, Eli Lilly and Company, and The Medicines Company, and has served as a consultant for EKR Pharmaceuticals, Baxter Healthcare, and The Medicines Company.

\section{References}

1. Pollack C Jr. RCJ. Hypertensive Emergencies: Acute Care Evaluation and Management. Emergency Medicine Cardiac Research and Education Group. 2008:3.

2. Chobanian AV, Bakris GL, Black HR, et al. The Seventh Report of the Joint National Committee on Prevention, Detection, Evaluation, and Treatment of High Blood Pressure: the JNC 7 report. JAMA. 2003;289(19):2560-2572.

3. Haas AR, Marik PE. Current diagnosis and management of hypertensive emergency. Semin Dial. 2006;19(6):502-512.

4. Ault MJ, Ellrodt AG. Pathophysiological events leading to the end-organ effects of acute hypertension. Am J Emerg Med. 1985;3 Suppl 6:10-15.

5. Majernick TG, Zacker C, Madden NA, Belletti DA, Arcona S. Correlates of hypertension control in a primary care setting. Am J Hypertens. 2004;17(10):915-920.

6. Marik PE, Varon J. Hypertensive crises: challenges and management. Chest. 2007;131(6):1949-1962.

7. Pitts WR, Lange RA, Cigarroa JE, Hillis LD. Cocaine-induced myocardial ischemia and infarction: pathophysiology, recognition, and management. Prog Cardiovasc Dis. 1997;40(1):65-76.

8. Catravas JD, Waters IW. Acute cocaine intoxication in the conscious dog: studies on the mechanism of lethality. J Pharmacol Exp Ther. 1981;217(2):350-356.

9. Stewart DL, Feinstein SE, Colgan R. Hypertensive urgencies and emergencies. Prim Care. 2006;33(3):613-623, v.

10. Karras DJ, Ufberg JW, Harrigan RA, Wald DA, Botros MS, McNamara RM. Lack of relationship between hypertension-associated symptoms and blood pressure in hypertensive ED patients. Am J Emerg Med. 2005;23(2):106-110. 
11. Borzecki AM, Wong AT, Hickey EC, Ash AS, Berlowitz DR. Hypertension control: how well are we doing? Arch Intern Med. 2003;163(22):2705-2711.

12. Pollack CV, Varon J, Garrison NA, Ebrahimi R, Dunbar L, Peacock WFt. Clevidipine, an intravenous dihydropyridine calcium channel blocker, is safe and effective for the treatment of patients with acute severe hypertension. Ann Emerg Med. 2009;53(3):329-338.

13. Prlesi L, Cheng-Lai A. Clevidipine: a novel ultra-short-acting calcium antagonist. Cardiol Rev. 2009;17(3):147-152.

14. Hussar DA. New drugs: Clevidipine butyrate, difluprednate, and tetrabenazine. J Am Pharm Assoc (2003). 2008;48(6):815-817, 819, 821.

15. The Medicines Company I. Cleviprex ${ }^{\mathrm{TM}}$ (clevidipine butyrate) prescribing information [online] [updated 2008 August; cited 25 August 2008]. Available from http://www.cleviprex.com/media/ClevidipinePI.pdf

16. Huraux C, Makita T, Szlam F, Nordlander M, Levy JH. The vasodilator effects of clevidipine on human internal mammary artery. Anesth Analg. 1997;85(5):1000-1004.

17. Ericsson H, Schwieler J, Lindmark BO, Lofdahl P, Thulin T, Regardh CG. Enantioselective pharmacokinetics of the enantiomers of clevidipine following intravenous infusion of the racemate in essential hypertensive patients. Chirality. 2001;13(3):130-134.

18. Ericsson H, Tholander B, Bjorkman JA, Nordlander M, Regardh CG. Pharmacokinetics of new calcium channel antagonist clevidipine in the rat, rabbit, and dog and pharmacokinetic/pharmacodynamic relationship in anesthetized dogs. Drug Metab Dispos. 1999;27(5):558-564.

19. Zhang JG, Dehal SS, Ho T, et al. Human cytochrome $\mathrm{p} 450$ induction and inhibition potential of clevidipine and its primary metabolite h152/81. Drug Metab Dispos. 2006;34(5):734-737.

20. Ericsson H, Bredberg U, Eriksson U, Jolin-Mellgard A, Nordlander M, Regardh CG. Pharmacokinetics and arteriovenous differences in clevidipine concentration following a short- and a long-term intravenous infusion in healthy volunteers. Anesthesiology. 2000;92(4): 993-1001.

21. Nordlander M, Sjoquist PO, Ericsson H, Ryden L. Pharmacodynamic, pharmacokinetic and clinical effects of clevidipine, an ultrashort-acting calcium antagonist for rapid blood pressure control. Cardiovasc Drug Rev. 2004;22(3):227-250.

22. Bailey JM, Lu W, Levy JH, et al. Clevidipine in adult cardiac surgical patients: a dose-finding study. Anesthesiology. 2002 96(5):1086-1094.

23. Gray RJ. Postcardiac surgical hypertension. J Cardiothorac Anesth. 1988;2(5):678-682.

24. Leung JM, O’Kelly BF, Mangano DT. Relationship of regional wall motion abnormalities to hemodynamic indices of myocardial oxygen supply and demand in patients undergoing CABG surgery. Anesthesiology. 1990;73(5):802-814.

25. Haas CE, LeBlanc JM. Acute postoperative hypertension: a review of therapeutic options. Am J Health Syst Pharm. 2004;61(16): 1661-1673; quiz 1674-1665.
26. Levy JH, Mancao MY, Gitter R, et al. Clevidipine effectively and rapidly controls blood pressure preoperatively in cardiac surgery patients: the results of the randomized, placebo-controlled efficacy study of clevidipine assessing its preoperative antihypertensive effect in cardiac surgery-1. Anesth Analg. 2007;105(4):918-925.

27. Singla N, Warltier DC, Gandhi SD, et al. Treatment of acute postoperative hypertension in cardiac surgery patients: an efficacy study of clevidipine assessing its postoperative antihypertensive effect in cardiac surgery-2 (ESCAPE-2), a randomized, double-blind, placebo-controlled trial. Anesth Analg. 2008;107(1):59-67.

28. Cheung AT. Exploring an optimum intra/postoperative management strategy for acute hypertension in the cardiac surgery patient. J Card Surg. 2006;21 Suppl 1:S8-S14.

29. Aronson S, Boisvert D, Lapp W. Isolated systolic hypertension is associated with adverse outcomes from coronary artery bypass grafting surgery. Anesth Analg. 2002;94(5):1079-1084, table of contents.

30. Koch-Weser J. The therapeutic challenge of systolic hypertension. N Engl J Med. 1973;289(9):481-483.

31. Powroznyk AV, Vuylsteke A, Naughton C, et al. Comparison of clevidipine with sodium nitroprusside in the control of blood pressure after coronary artery surgery. Eur J Anaesthesiol. 2003; 20(9):697-703.

32. Estafanous FG, Tarazi RC. Systemic arterial hypertension associated with cardiac surgery. Am J Cardiol. 1980;46(4):685-694.

33. Aronson S, Dyke C, Stierer K, et al. The ECLIPSE Trials: Comparative Studies of Clevidipine to Nitroglycerin, Sodium Nitroprusside, and Nicardipine for Acute Hypertension Treatment in Cardiac Surgery Patients. Anesth Analg. 2008;107(4):ePub ahead of print.

34. Noviawaty I, Uzun G, Qureshi AI. Drug evaluation of clevidipine for acute hypertension. Expert Opin Pharmacother. 2008;9(14): 2519-2529.

35. Cada DJe, Levien TL, Baker DE. Clevidipine Butyrate Injectable Emulsion. Hospital Pharmacy. 2008;43(11):903-912.

36. Kenyon KW. Clevidipine: an ultra short-acting calcium channel antagonist for acute hypertension. Ann Pharmacother. 2009;43(7): 1258-1265.

37. Segawa D, Sjoquist PO, Wang QD, Gonon A, Ryden L. Time-dependent cardioprotection with calcium antagonism and experimental studies with clevidipine in ischemic-reperfused pig hearts: Part II. J Cardiovasc Pharmacol. 2002;40(3):339-345.

38. Gourine AV, Pernow J, Poputnikov DM, Sjoquist PO. Calcium antagonist clevidipine reduces myocardial reperfusion injury by a mechanism related to bradykinin and nitric oxide. J Cardiovasc Pharmacol. 2002;40(4):564-570.

39. Gourine A, Gonon A, Sjoquist PO, Pernow J. Short-acting calcium antagonist clevidipine protects against reperfusion injury via local nitric oxide-related mechanisms in the jeopardised myocardium. Cardiovasc Res. 2001;51(1):100-107.
Integrated Blood Pressure Control

\section{Publish your work in this journal}

Integrated Blood Pressure Control is an international, peer-reviewed open-access journal focusing on the integrated approach to managing hypertension and risk reduction. Treating the patient and comorbidities together with diet and lifestyle modification and optimizing healthcare resources through a multidisciplinary team approach constitute key

\section{Dovepress}

features of the journal. This journal is indexed on American Chemical Society's Chemical Abstracts Service (CAS). The manuscript management system is completely online and includes a very quick and fair peerreview system, which is all easy to use. Visit http://www.dovepress.com/ testimonials.php to read real quotes from published authors. 